\title{
Molecular Weight Effect on Local Motion of Polystyrene Studied by the Fluorescence Depolarization Method
}

\author{
Jun-ichi Horinaka, Hiroyuki Aoki, Shinzaburo Ito, and Masahide Yamamoto* \\ Department of Polymer Chemistry, Graduate School of Engineering, Kyoto University, \\ Sakyo, Kyoto 606-8501, Japan
}

(Received August 31, 1998)

\begin{abstract}
The molecular weight effect on the local motion of polystyrene (PS) was examined in dilute solutions by the fluorescence depolarization method. Four PS samples with the fluorescent probe, anthryl group, in the middle of the main chain were synthesized by the living anionic polymerization. The molecular weight of samples varied from $c a .6 .4 \times 10^{3}$ to $9.2 \times 10^{4}$. Solvents were benzene, a good solvent and ethyl acetate, a poor solvent. In both solvents, the relaxation time increased with the molecular weight up to $\mathrm{MW}=10^{4}$ at which it reached an asymptotic value. The activation energies were also estimated from the temperature dependence of the relaxation time, and its molecular weight dependence appeared to be similar to that of the relaxation time. It was suggested that the relaxation time of the local motion is determined by the potential for the conformational transition of main chain bonds, rather than by the segment density. Finally, the molecular weight effect on the relaxation time for PS was compared with that for poly(oxyethylene) (POE). The results showed that PS is dynamically stiffer than POE.

KEY WORDS Molecular Weight Effect / Local Chain Motion / Polystyrene / Fluorescence Depolarization / Segment Density / Potential Energy / Chain Stiffness /
\end{abstract}

The flexible polymer chain in dilute solution has various motional scales with regard to time and space resulting from its high degree of intramolecular freedom. Because this chain dynamics governs a variety of properties of polymers, extensive experimental and theoretical efforts have been made to understand the polymer chain dynamics. ${ }^{1-21}$ For the local motion, which is fairly a fundamental process in chain dynamics, many experimental methods have been utilized, e.g., NMR, ${ }^{5-7}$ $\mathrm{ESR},{ }^{8}$ dielectric relaxation, ${ }^{9-11}$ dynamic light scattering, ${ }^{12,13}$ neutron scattering, ${ }^{14}$ and fluorescence depolarization. ${ }^{15-21}$ The fluorescence depolarization method provides direct information about the local motion of polymer chains through a fluorescent probe that is covalently bonded to the polymer main chain. By using this method, we have examined the influence of molecular structure, ${ }^{16,19}$ stereoregularity, ${ }^{17}$ and quality of solvent $^{16,18}$ on the chain dynamics of a variety of polymers.

The local chain dynamics in dilute solution is influenced by the molecular weight of the polymer in addition to those factors mentioned above. Concerning the fluorescence depolarization study, Waldow et al. have examined the molecular weight effect on polyisoprene (PI) chain dynamics and concluded that the chain dynamics is governed by the segment density in the vicinity of the fluorescent probe labeled in the middle of the main chain. ${ }^{21}$ Previously, we reported the molecular weight effect for poly(methyl methacrylate) samples and explained the behavior of the local chain dynamics by the segment density as well. ${ }^{19} \mathrm{We}$ also have reported the local chain dynamics of poly(oxyethylene) (POE) in good solvents and discussed the molecular weight effect. $^{20}$

The static and dynamic properties of polystyrene (PS), a common polymer, have been widely studied. ${ }^{2,6-9,16,17}$ We have reported the effects of the solvent quality on the local dynamics of PS $^{17}$ and discussed the difference

To whom correspondence should be addressed. of the chain mobility for styrene derivative polymers. ${ }^{16}$ From the standpoint of the molecular structure, it is interesting to compare the molecular weight effect of PS with that of POE, which is dynamically more flexible than PS. ${ }^{20}$

Monnerie et al. studied the molecular weight effect of PS on the local motion by $\mathrm{NMR},{ }^{6}$ and concluded that the effect of the overall rotational diffusion is negligible on the spin-spin relaxation time of the local motion in a high molecular weight region and that the correlation time remains constant in $\mathrm{MW}>10^{4}$. Prior to Monnerie's work, similar findings have been reported by Allerhand and Hailstone, ${ }^{7}$ Stockmayer and Matsuo, ${ }^{9}$ and Bullock et al. ${ }^{8}$ They all concluded that at high molecular weights the relaxation time of local motion is independent of chain length, while as the molecular weight decreases the end-over-end rotation contributes to the relaxation process.

In the present study, we examined the local motion of four PS samples in dilute solution by the fluorescence depolarization method. PS samples were labeled in the middle of the main chain with the anthryl group. We examined the molecular weight effect on the chain dynamics of PS in two solvents, and also compared the molecular weight effect for PS with that for POE.

\section{EXPERIMENTAL}

\section{Sample Preparation}

Anthryl group-labeled PS samples used in this study were synthesized by the living anionic polymerization in vacuo initiated by butyllithium, in which the living ends were coupled with 9,10-bis(bromomethyl)anthracene. The polymerization was carried out at $40^{\circ} \mathrm{C}$ (except cPS92) in benzene, which was dried over sodium. Styrene monomer (Nacalai Tesque) was dried over fresh calcium hydride after distillation. The coupling reaction was carried out at $c a .5^{\circ} \mathrm{C}$. The coupler, 9,10-bis(bromomethyl)anthracene, was dissolved in dried tetrahydro- 


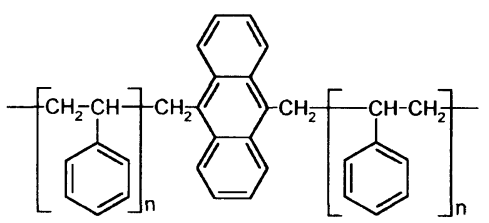

Figure 1. Molecular structure of the PS sample labeled with anthryl group in the middle of the main chain.

Table I. Characterization of polystyrene samples used in this study

\begin{tabular}{lccc}
\hline Sample & $M_{w} \times 10^{-4}$ & $M_{n} \times 10^{-4}$ & $M_{w} / M_{n}$ \\
\hline cPS92 & 9.7 & 9.2 & 1.05 \\
cPS69 & 7.2 & 6.9 & 1.04 \\
cPS13 & 1.39 & 1.31 & 1.06 \\
cPS6 & 0.72 & 0.64 & 1.13 \\
\hline
\end{tabular}

furan before use. Further details of procedure appear elsewhere. ${ }^{22}$ The product polymer was purified by reprecipitation from benzene in methanol. The incompletely coupled fraction was removed from the original sample by GPC. The weight-average molecular weight $M_{w}$ and the number-averaged molecular weight $M_{n}$ of fractionated PS were determined by GPC. Figure 1 and Table I show the molecular structure and the characterization of PS samples, respectively. cPS92 was the same sample used in previous studies. ${ }^{16,17}$ The molecular weight distribution of all the samples was sufficiently narrow.

Solvents used in measurements were benzene (Dojin, spectrophotometric grade) and ethyl acetate (Nacalai Tesque, spectrosol). Both were used without further purification. The quality of these solvents for PS was obtained by the intrinsic viscosity measurement. ${ }^{17}$ Benzene $\left(\bar{\alpha}_{\eta}^{3}=1.66\right)$ was a better solvent than ethyl acetate $\left(\bar{\alpha}_{\eta}^{3}=1.12\right)$. In preparing the sample solutions, each polymer concentration was kept less than $10^{-5} \mathrm{M}$. Each solution was put into a quartz cell and degassed.

\section{Anisotropy Decay Measurement}

The measurement of time-resolved anisotropy decay was carried out by the single photon counting system. ${ }^{20}$ The second harmonic of Ti:sapphire laser (SpectraPhysics Tsunami) was used at a wavelength of $397 \mathrm{~nm}$ as a light source and $4 \mathrm{MHz}$ pulse was picked up by a pulse selector (Spectra-Physics Model 3980). The excitation light was vertically polarized, and the fluorescence components parallel and perpendicular to the plane of the excitation light were measured by a microchannel plate-photomultiplier tube (MCP-PMT) (Hamamatsu Photonics R3809) through cutoff filters V-42 and Y-44 (Hoya). The signal of MCP-PMT was inputted to a time-to-amplitude converter (Ortec Model 457) as a start signal through a constant fraction discriminator (Ortec Model 583). The stop signal was the fundamental laser beam detected by a photo-diode (Antel Optronics AR-S2). The time-resolved fluorescence intensity was analyzed by a multichannel analyzer (Norland Ino-Tech 5300). The FWHM of the total instrumental function was $c a$. $60 \mathrm{ps}$. The parallel component, $I_{\mathrm{Vv}}(t)$, and the perpendicular component, $I_{\mathrm{VH}}(t)$, were measured alternatively to avoid data distortions

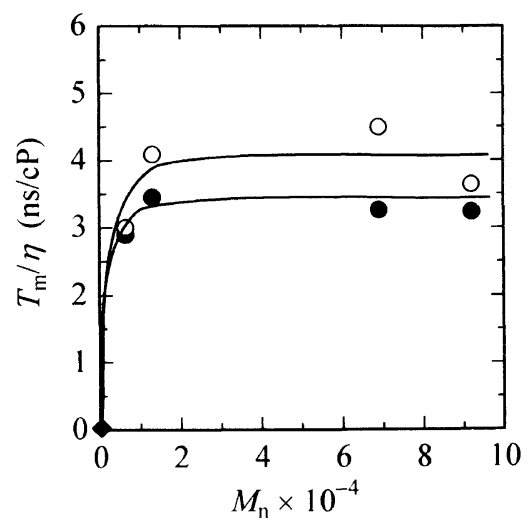

Figure 2. Molecular weight dependence of the reduced relaxation time $T_{\mathrm{m}} / \eta$ for PS at $20^{\circ} \mathrm{C}$ : (O) in benzene; $(\bigcirc)$ in ethyl acetate. Filled diamond $(\bullet)$ represents the rotational relaxation time of DMA, the probe molecule. The relaxation time reaches an asymptotic value at $\mathrm{MW} \cong 10^{4}$.

due to the time drift. Anisotropy decay measurements were carried out in the temperature range from $c a .10$ to $30^{\circ} \mathrm{C}$.

\section{Data Analysis}

The fluorescence anisotropy ratio, $r(t)$, is defined as

$$
r(t)=\left(I_{\mathrm{VV}}(t)-G I_{\mathrm{VH}}(t)\right) /\left(I_{\mathrm{VV}}(t)+2 G I_{\mathrm{VH}}(t)\right)
$$

where $G$ is the compensating factor. We estimated $G$ to be unity in this study. For discussion about the chain mobility, we use the integrated relaxation time, $T_{\mathrm{m}}$, which is defined as eq 2. $T_{\mathrm{m}}$ represents the average value of the relaxation times.

$$
T_{\mathrm{m}}=r_{0}^{-1} \int_{0}^{\infty} r(t) \mathrm{d} t
$$

where $r_{0}$ is the initial anisotropy ratio.

\section{RESULTS AND DISCUSSION}

\section{Relaxation Time}

Figure 2 shows the molecular weight dependence of the reduced relaxation time $T_{\mathrm{m}} / \eta$ for PS in benzene and in ethyl acetate at $20^{\circ} \mathrm{C}$. We used benzene as a good solvent and ethyl acetate as a poor solvent in this study. Note that the relaxation time $T_{\mathrm{m}}$ was reduced by the solvent viscosity $\eta$ according to the theory of Kramers' diffusion limit. The solvent viscosity was estimated from the values reported in the literature $25: 0.65 \mathrm{cP}$ for benzene and $0.45 \mathrm{cP}$ for ethyl acetate at $20^{\circ} \mathrm{C}$. The solvent viscosity dependence of the relaxation time was discussed repeatedly in our previous studies. ${ }^{20,26-32}$ Consequently, we know that the relaxation time for the local motion of PS labeled in the middle of the main chain in a low viscosity solvent below $1 \mathrm{cP}$ is inversely proportional to the solvent viscosity, i.e., the Kramers' theory holds. The formulation of Kramers' theory is given in the following subsection. The filled diamond in Figure 2 indicates the reduced rotational relaxation time of the model compound, 9,10-dimethylanthracene (DMA), in benzene at $20^{\circ} \mathrm{C}$ estimated by the steady-state measurement. The rotational relaxation time for DMA can be regarded as the limiting value of low molecular weight PS. Figure 2 
shows that $T_{\mathrm{m}} / \eta$ in each solvent increases with molecular weight and reaches an asymptotic value at about $\mathrm{MW}$ of $10^{4}$. This indicates that the local motion, which contributes to the fluorescence depolarization, occurs within the scale of $\mathrm{MW}=10^{4}$. In other words, the local motion for PS of a higher molecular weight is independent of the entire rotational motion.

Waldow et al. showed that the relaxation time for the local motion of the chain center of PI does not change with molecular weight in a good solvent, whereas the relaxation time increases with molecular weight in a $\Theta$ solvent. ${ }^{21}$ They explained these observations by the local segment concentration about the labeled segment. That is, the molecular weight independence of the relaxation time in a good solvent is due to the excluded-volume effect, which tends to keep segments which are far apart along the chain contour far separated in space. In a $\Theta$ solvent, where the excluded-volume effect is almost canceled, the local concentration increases with molecular weight. In the case of POE, the result that the relaxation time in a good solvent was kept constant against molecular weight in a high molecular weight region was similarly explained by the excluded-volume effect. ${ }^{20}$ However in this study, the relaxation time in ethyl acetate (poor solvent) was almost constant against molecular weight as in benzene (good solvent) in a wide range of high molecular weight, i.e., the values of $T_{\mathrm{m}} / \eta$ near $\mathrm{MW}=10^{5}$ are almost equal to that for cPS13. Ethyl acetate is a poor solvent, and the coil expansion due to excluded-volume effect for high molecular weight PS is considered to be rather weak. Then the segment density of each molecular weight sample would be different, but this is not the case.

Abe et al. have studied the excluded-volume effects on the mean-square radius of gyration $\left\langle S^{2}\right\rangle$ and on the intrinsic viscosity $[\eta]$ of oligo- and polystyrenes in dilute solutions. ${ }^{2,23,24}$ The values of $\left\langle S^{2}\right\rangle$ and $[\eta]$ for atactic-PS (a-PS) in toluene at $15^{\circ} \mathrm{C}$ were compared with those in cyclohexane at $34.5^{\circ} \mathrm{C}(\Theta)$ in the range of $M_{w}$ from $5.78 \times 10^{2}$ to $3.84 \times 10^{6}$. They used the oligomer samples to realize an unperturbed state without intramolecular excluded-volume interaction even in a good solvent. In practice, they chose the solvent (toluene) and temperature $\left(15^{\circ} \mathrm{C}\right)$ so that the unperturbed dimension of the PS chain in a good solvent may coincide with that in the $\Theta$ condition taken as a reference standard. It was shown that, for the weight-average degree of polymerization $x_{w}<20$, the data of both $\left\langle S^{2}\right\rangle$ and $[\eta]$ in toluene at $15^{\circ} \mathrm{C}$ are in good agreement with those in cyclohexane at $\Theta$ temperature. This agreement implies that the dimensions and conformations of the a-PS chain in the unperturbed state is considered the same under the two solvent conditions. For large $x_{w}$, the data points for the toluene solutions deviate progressively from those for the cyclohexane solutions with increasing $x_{w}$, clearly due to the excluded-volume effect. Their data show that the intramolecular interaction at $M_{w}=10^{5}$ is sufficiently larger than that at $M_{w}=10^{4}$, which leads to the difference in the segment density in the case of no or weak excluded-volume effect. Consequently, the segment density cannot explain the behavior of $T_{\mathrm{m}} / \eta$ in this study, because the relaxation time is almost constant in the high molecular weight region even in a poor solvent. Then, we will propose another reason. The local motion consists of the conformational transition over the potential barrier and the librational motion within the potential well. ${ }^{34}$ Therefore, there is a possibility that the local potential energy for the conformational transition of the main chain bond is affected by the solvent condition as well as by the molecular structure of each segment. The potential energy is a local factor compared with the segment density, so that it has no relation with the excluded-volume effect between the segments far apart.

Now let us discuss the difference in the asymptotic value of $T_{\mathrm{m}} / \eta$ and in the critical molecular weight between two solvents: good and poor solvents. We see from Figure 2 that the value of $T_{\mathrm{m}} / \eta$ for ethyl acetate solution is larger than that for benzene solution at each molecular weight. Figure 2 also shows that the critical molecular weight, $M_{\mathrm{c}}$, at which $T_{\mathrm{m}} / \eta$ reaches its asymptotic value, is slightly larger in ethyl acetate. As mentioned above, it is considered that the difference between two solvents comes from the difference in the potential energy for the local conformational transition due to the degree of chain expansion with the solvent quality. It is noteworthy that the difference in $T_{\mathrm{m}} / \eta$ is observed even for low molecular weight PS samples, cPS13 and cPS7. Abe et al. showed that the difference between the values of $\left\langle S^{2}\right\rangle$ or $[\eta]$ in the two solvents is almost negligible below $x_{w}$ of $10^{2}$ $\left(M_{w}=10^{4}\right)$. So PS of $\mathrm{MW} \cong 10^{4}$ can be considered nearly an unperturbed chain that is little influenced by the excluded-volume effect. The fact that the difference in $T_{\mathrm{m}} / \eta$ between solvents was observed even for nearly an unperturbed chain supports the proposition that the potential energy is the main governing factor of the local motion. The local potential energy is considered to affect the local motion even in the low molecular weight region. Although the relation between local potential energy and solvent condition is unclear, we assume that the poorer the solvent condition the higher the potential energy. The studies on the local motion of oligo- and polystyrene chain ends which supports this explanation more definitely will be reported separately. ${ }^{33}$

The objection may be raised that our scheme supposing the difference in the potential energy between two solvents, in benzene and in ethyl acetate, is inconsistent with Abe's findings that the dimensions and conformations may be the same under the two solvent conditions in $x_{w}<20$. This can be explained by the fact that the unperturbed chain dimension is not independent of solvent and temperature. That is, in our case, it is possible that the chain dimension is slightly different between the two solvents. Therefore, the difference in the value of the relaxation time and in its molecular weight dependence may be due to the difference in the local potential energy for the conformational transition of the main chain bond. In addition, the dynamic properties are influenced by both the value of energy potential minimum and the energy barrier height, while the static properties are influenced by the former.

We have some comments on previous works. Our suggestion in this study does not intend to deny the concept of the segment density proposed by Waldow et $a l .{ }^{21}$ In our previous study, it was shown that PI is dynamically more flexible than PS. ${ }^{17}$ Therefore, the 


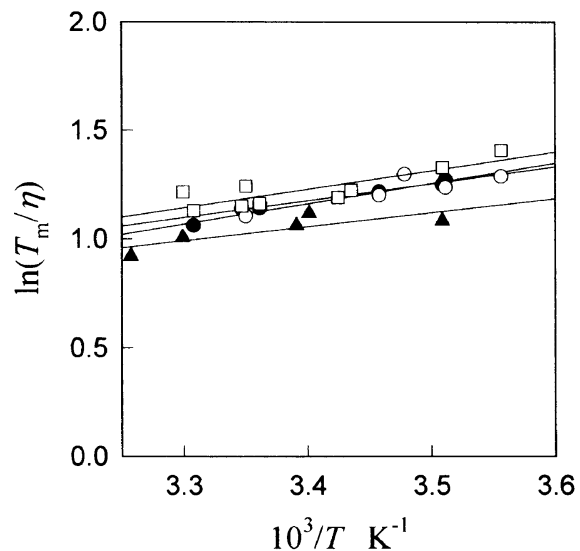

(a)

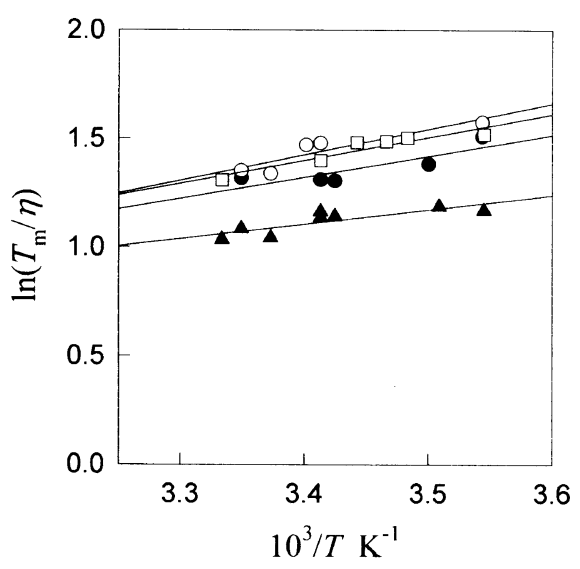

(b)

Figure 3. $\ln \left(T_{\mathrm{m}} / \eta\right)$ vs. $1 / T$ plot according to the theory of Kramers' diffusion limit. $T_{\mathrm{m}}$ was measured (a) in benzene, and (b) in ethyl acetate: (О) cPS92; (○) cPS69; ( $\square$ ) cPS13; ( $\mathbf{\Delta})$ cPS6.

excluded-volume effect for PI may be more effective than that for PS and the local motion is mainly determined by the segment density in the high molecular weight region.

\section{Activation Energy}

Next, we estimated the activation energy of the local motion according to the theory of Kramers' diffusion limit as follows. ${ }^{1}$ Here the formulation of Kramers' theory is given. The velocity coefficient, $k$, of a particle with a frictional coefficient, $\zeta$, passing over an energy barrier of the height $E$ is represented as

$$
k \propto \zeta^{-1} \exp (-E / R T),
$$

where $R$ is the gas constant and $T$ is absolute temperature. The fact that $T_{\mathrm{m}}$ is proportional to the reciprocal of $k$ and the solvent viscosity, $\eta$, is proportional to $\zeta$, according to Stokes' law, leads to

$$
T_{\mathrm{m}} / \eta=A \exp \left(E^{*} / R T\right) .
$$

Figure 3 shows a temperature dependence of $T_{\mathrm{m}}$ for PS, (a) in benzene, and (b) in ethyl acetate solvents. The relaxation time $T_{\mathrm{m}} / \eta$, which was reduced by the solvent viscosity, $\eta$, was plotted according to the theory of Kramers' diffusion limit so that the activation energies could be estimated from the slope of the plot in Figure 3.

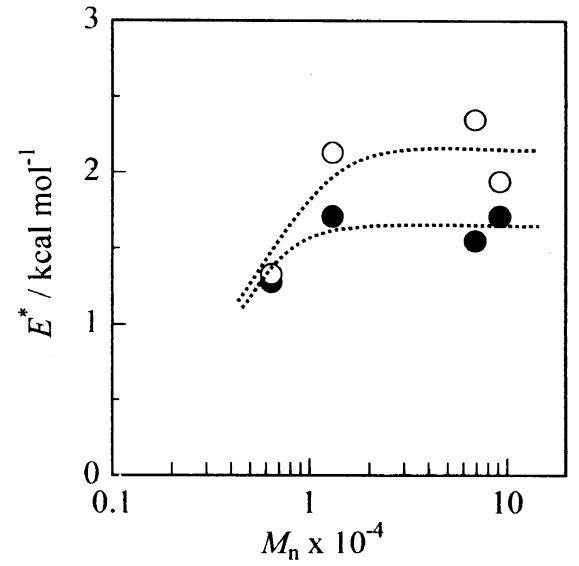

Figure 4. Molecular weight dependence of the activation energy $E^{*}$ for PS estimated from the slope of the Arrhenius plot in Figure 3(a) and $(b)$ : $(O)$ in benzene; $(O)$ in ethyl acetate.

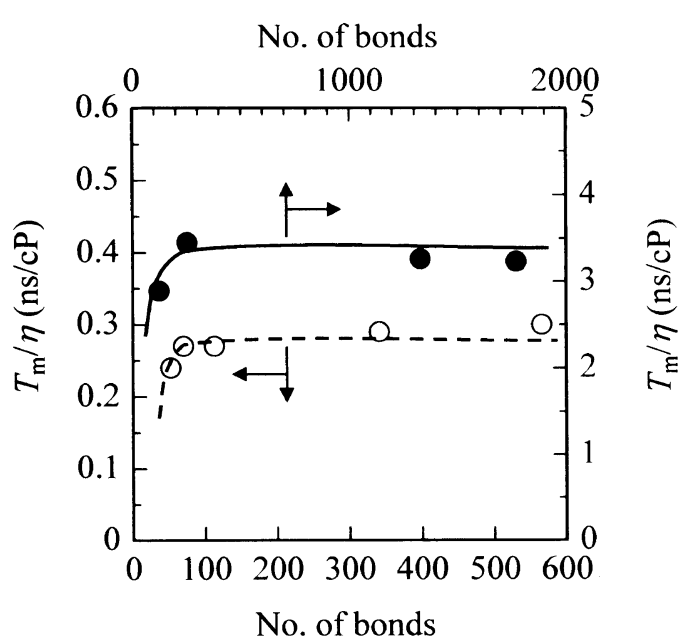

Figure 5. Relationship between the reduced relaxation time $T_{\mathrm{m}} / \eta$ and the number of bonds for PS in benzene (O) at $20^{\circ} \mathrm{C}$ and POE in DMF (A) at $13^{\circ} \mathrm{C}$. The critical points, $N_{\mathrm{c}}$, for PS and POE are about 200 and 70 , respectively.

Figure 4 shows the relationship between the molecular weight and the estimated activation energy in benzene. The activation energy as well as the relaxation time tends to increase with molecular weight and to become constant at $\mathrm{MW} \cong 10^{4}$. The fact that the activation energy is constant in the high molecular weight region indicates that the mode of local motion is the same in the region. Figure 4 also shows that the activation energy in ethyl acetate is larger than that in benzene. This is due to the difference in the energy potential for the conformational transition.

\section{Chain Length of Cooperative Motion}

Finally, we compare the molecular weight effect on the local motion of PS with that of $\mathrm{POE}^{20}$ and discuss the critical number of bonds, $N_{\mathrm{c}}$, at which the relaxation time becomes constant. It is considered that the cooperative local motion of polymer chain operates up to this critical number of bonds and this value may indicate a kind of dynamic chain stiffness. Figure 5 shows the plots of $T_{\mathrm{m}} / \eta v s$. number of bonds for both PS at $20^{\circ} \mathrm{C}$ and POE at $13^{\circ} \mathrm{C}$ in each good solvent. It shows that the value of reduced relaxation time for PS is larger 
than that for POE. For PS, this is due to the large substituent attached to the main chain, the phenyl ring. ${ }^{20}$ Here, we compared $N_{\mathrm{c}}$ between PS and POE. We estimated $N_{\mathrm{c}}$ for POE to be ca. 70, while for PS, $N_{\mathrm{c}}$ is 200. That is, PS is dynamically stiffer than POE. The chain length of cooperative motion reflects the molecular structure of the monomer unit and closely correlates with the absolute value of the relaxation time above $N_{\mathrm{c}}$.

\section{CONCLUSION}

The molecular weight effect on the local motion of PS labeled in the middle of the main chain in dilute solution was studied by the fluorescence depolarization method. Four samples in the molecular weight from $c a .6 .4 \times$ $10^{3}$ to $9.2 \times 10^{4}$, were synthesized by living anionic polymerization. Benzene was used as a good solvent, while ethyl acetate was used as a poor solvent. The relaxation time as well as the activation energy increased with molecular weight up to $\mathrm{MW}$ of $10^{4}$ and reached an asymptotic value in both solvents. The critical molecular weight, $M_{\mathrm{c}}$, corresponds to the largest size unit in the local motion measured by the fluorescence depolarization method. The difference in the relaxation time and molecular weight dependence between two solutions may result from the local potential for the conformational transition of main chain bond, rather than the segment density. The critical chain length of the local motion, which indicates a kind of the dynamic chain stiffness, of PS is larger than that of POE.

Acknowledgments. We would like to thank Dr. Takashi Sasaki, Fukui University, for the preparation and the synthetic technique of the anthryl group-labeled PS. This work was supported by a Grant-in-Aid for Scientific Research (No. 09450359) from the Ministry of Education, Science, Sports and Culture of Japan and was partly supported by a Research Fellowship of the Japan Society for the Promotion of Science for Young Scientists.

\section{REFERENCES}

1. E. Helfand, J. Chem. Phys., 54, 4651 (1971).

2. H. Yamakawa, "Helical Wormlike Chains in Polymer Solutions," Springer-Verlag, Berlin, 1997.

3. J. D. Ferry, "Viscoelastic Properties of Polymers," 3rd ed, Wiley \& Sons, New York, N.Y., 1980.
4. M. Doi and S. F. Edwards, "The Theory of Polymer Dynamics," Clarendon Press, Oxford, 1986.

5. S. Glowinkowski, D. J. Gisser, and M. D. Ediger, Macromolecules, 23, 3520 (1990)

6. F. Lauprêtre, C. Noël, and L. Monnerie, J. Polym. Sci., Polym. Phys. Ed., 15, 2127 (1977).

7. A. Allerhand and R. K. Hailstone, J. Chem. Phys., 77, 1635 (1972).

8. A. T. Bullock, G. G. Cameron, and P. M. Smith, J. Phys. Chem., 77, 1635 (1973).

9. W. H. Stockmayer and K. Matsuo, Macromolecules, 5, 766 (1972).

10. S. Mashimo, P. Winsor, R. H. Cole, K. Matsuo, and W. H. Stockmayer, Macromolecules, 19, 682 (1986).

11. K. Adachi, Macromolecules, 23, 1816 (1990).

12. T. Yoshizaki and H. Yamakawa, J. Chem. Phys., 99, 9145 (1993).

13. Y. Takaeda, T. Yoshizaki, and H. Yamakawa, Macromolecules, 27, 4248 (1994).

14. T. Kanaya, K. Kaji, and K. Inoue, Macromolecules, 24, 1826 (1991).

15. T. Sasaki and M. Yamamoto, Macromolecules, 22, 4009 (1989).

16. K. Ono, Y. Okada, S. Yokotsuka, T. Sasaki, S. Ito, and M. Yamamoto, Macromolecules, 27, 6482 (1994).

17. K. Ono, K. Ueda, T. Sasaki, S. Murase, and M. Yamamoto, Macromolecules, 29, 1584 (1996).

18. K. Ono, T. Sasaki, M. Yamamoto, Y. Yamasaki, K. Ute, and K. Hatada, Macromolecules, 28, 5012 (1995).

19. J. Horinaka, K. Ono, and M. Yamamoto, Polym. J., 27, 429 (1995).

20. J. Horinaka, S. Amano, H. Funada, S. Ito, and M. Yamamoto, Macromolecules, 31, 1197 (1998).

21. D. A. Waldow, B. S. Johnson, P. D. Hyde, M. D. Ediger, T. Kitano, and K. Ito, Macromolecules, 22, 1345 (1989).

22. T. Sasaki, M. Yamamoto, and Y. Nishijima, Makromol. Chem., Rapid Commun., 7, 345 (1986).

23. F. Abe, Y. Einaga, T. Yoshizaki, and H. Yamakawa, Macromolecules, 26, 1884 (1993)

24. F. Abe, Y. Einaga, H. Yamakawa, Macromolecules, 26, 1891 (1993).

25. J. A. Riddick and W. B. Bunger, "Techniques of Chemistry II, Organic Solvents," 3rd ed, Wiley-Interscience, New York, N.Y., 1970.

26. D. B. Adolf, M. D. Ediger, T. Kitano, and K. Ito, Macromolecules, 25, 867 (1992).

27. S. Glowinkowski, D. J. Gisser, and M. D. Ediger, Macromolecules, 23, 3520 (1990)

28. K. Ono, Y. Okada, S. Yokotsuka, S. Ito, and M. Yamamoto, Polym. J., 26, 199 (1994).

29. S. P. Velsko and G. R. Fleming, J. Chem. Phys., 76, 3553 (1982).

30. S. P. Velsko, D. H. Waldeck, and G. R. Fleming, J. Chem. Phys., 78, 249 (1983).

31. R. F. Grote and J. T. Hynes, J. Chem. Phys., 73, 2715 (1980).

32. B. Bagchi and D. W. Oxtoby, J. Chem. Phys., 78, 2735 (1983).

33. J. Horinaka, S. Ito, and M. Yamamoto, Macromolecules, submitted.

34. G. J. Moro, J. Chem. Phys., 97, 5749 (1992). 